\title{
Research on image mosaictechnique based on Contourlet transform
}

\author{
LI LI ${ }^{1 \text {, a }}$, Chen Jun bo ${ }^{2, a}$, Cheng Jin ${ }^{3, a}$ \\ ${ }^{1}$ WuhanUniversity of technology huaxia college Wuhan,430223China \\ ${ }^{2}$ Wuhan university school of power and mechanical ,automation \\ majorWuhan,430072China \\ ${ }^{3}$ WuhanUniversity of Technology,Wuhan,430070,China \\ aemail:176527785@qq.com
}

\begin{abstract}
The image after image mosaic apply in not demanding occasions case, This paper presents the splicing method based on Contourlet transform.Through the feature extraction, feature matching, ranking is obtainedafter image stitching. The experimental results show that this method improves the quality of image stitching.

Keywords: Stitching; Image Registration; Image Fusion

\section{Introduction}

In recent years, image stitching technology of [1] in daily life are everywhere. Such as access to a clear cover larger size of the image, the ordinary camera can not meet the requirements, the wide-angle lens or panoramiccamera is not only expensive and use method is relatively complex, andthe edge of the wide-angle lens will produce distortion phenomenon.
\end{abstract}

\section{Contourlet transform}

The dominant idea of Contourlet transform of is through Laplacian pyramid decomposition is referred to as LP and direction (Directional Filter Bank) DFB filter to achieve. Compared to the past, those of an ordinary method, it is directed to a method of discrete images, can also achieve the multi-scale, multi direction of the frame. As shown in Figure 1 is a dual filter bank structure,First, according to the decomposition of LP transform Pyramid [2] take on multi-scale image, after obtaining the point singularity, then the high frequency components of each level in front of the Pyramid to take direction filter, finally using directional filter DFB, the singular point the same direction are combined into a coefficient. This completes the Contourlet transform. 


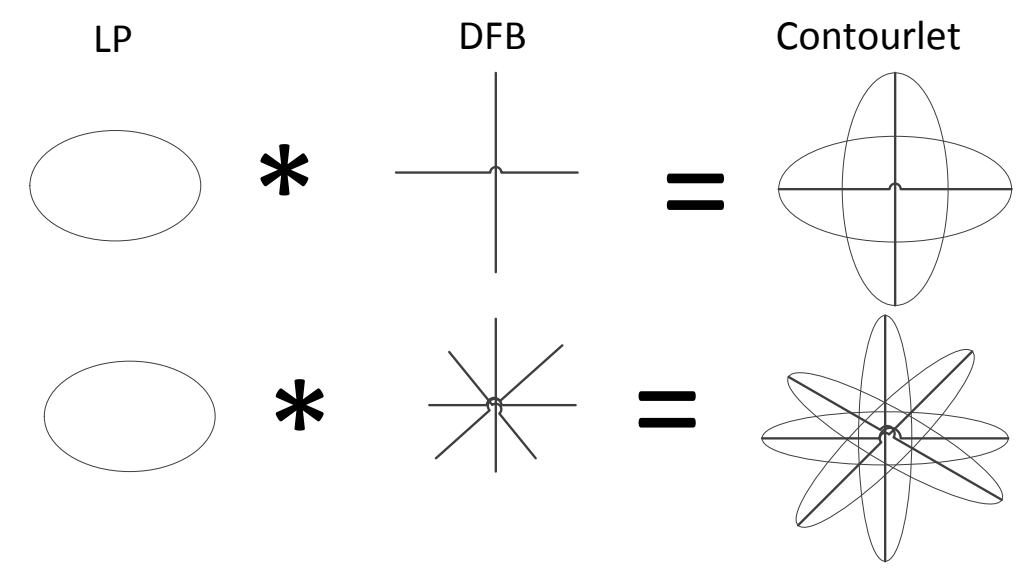

Fig.1Contourlet transform briefly the implementation process

\section{Image matching}

\subsection{Image feature point detection}

This paper uses Harris corner [8] feature point detection algorithm. assuming the pixel coordinate value of $(\mathrm{x}, \mathrm{y})$, hypothesis testing window is $\mathrm{w}$, the w mobile $\mathrm{u}$ in the $\mathrm{X}$ direction, the mobile $\mathrm{V}$ in the $\mathrm{Y}$ direction, Harris gray scale change in the analytic formula for the:

$$
\mathrm{E}_{\mathrm{x}, \mathrm{y}}=\sum W_{x, y}\left(I_{x+u, y+v}-I_{x, y}\right)^{2}
$$

Wherein, I represents a window function. W (x, y) represent the grayfunction, which is defined as: $W_{x, y}=e^{-\left(x^{2}+y^{2}\right) / \delta^{2}}$, The formula (1) can be obtained after treatment:

$$
E_{x, y}=R^{-1}\left[\begin{array}{cc}
\lambda_{1} & 0 \\
0 & \lambda_{2}
\end{array}\right] R(2)
$$

Among them, $\mathrm{R}$ said the rotation factor and its characteristic value of curvature representation in spindle orientation value. If the eigen value sare very small, show that near here is "flat area", if the eigen value size disparity shows the feature points in the image edge. If the feature values are not poor, but the eigen values are large, no matter which direction to move along will make dramatic changes into the gray, the expression Harris corner point:

$$
\mathrm{CRF}(\mathrm{x}, \mathrm{y})=\operatorname{det}(\mathrm{M})-\mathrm{k}(\operatorname{trace}(\mathrm{M}))^{2}(3)
$$

Among them, said M matrix, DET (M) said the determinant of $\mathrm{M}$, trace (M)represents the trace of a matrix, $\mathrm{K}$ said a predetermined threshold,typically $\mathrm{K}$ between $0.04 \sim 0.06$. To obtain the objective of CRF is greater than a given threshold value $\mathrm{K}$, then the pixel is the image of the corner. 


\subsection{Image matching}

Institutions Opt Image matching idea is: first the feature extraction (feature points and feature lines, regional characteristics, this paper uses feature points).The feature extraction of the coarse matching and fine matching.

(1) The coarse matching

It uses BBF algorithm to get the nearest neighbor and next nearest neighbor points, and then get the ratio of nearest neighbor feature points of the Euclidean distance between D1 and the next nearest neighbor points of the Euclidean distance between D2 h, if $\mathrm{h}$ is less than the set threshold (the 0.6 ) is to determine whether the two feature points are matched.

(2) Fine matching

The coarse matching the above income usually exist mismatches, require purification with higher accuracy on matching as the final matching further, called fine matching, this phase is completed by [3] RANSAC method.

(3) Solving the transformation matrix

Two in the original image overlap a point part, can be seen as the pointfrom different angle shooting, At this time, there is the original image transform matrix between the coordinate. Will the two images stitching together, You will need according to the matrix will be a projection of the image to another image, Complete the image integration. $T$ his article take solving perspective transformation model to realize the transformation matrix.

\subsection{Analysis of experimental results}

The following to mosaic of two images as an example to carry on the experiment, the concrete steps and results are as follows:

1.Image feature point extraction, Feature extraction results are shown in Figure 2.

2.The coarse matching, Image coarse matching results shown as in Figure 3.

3.Fine matching, Image matching results are shown in Figure 4.

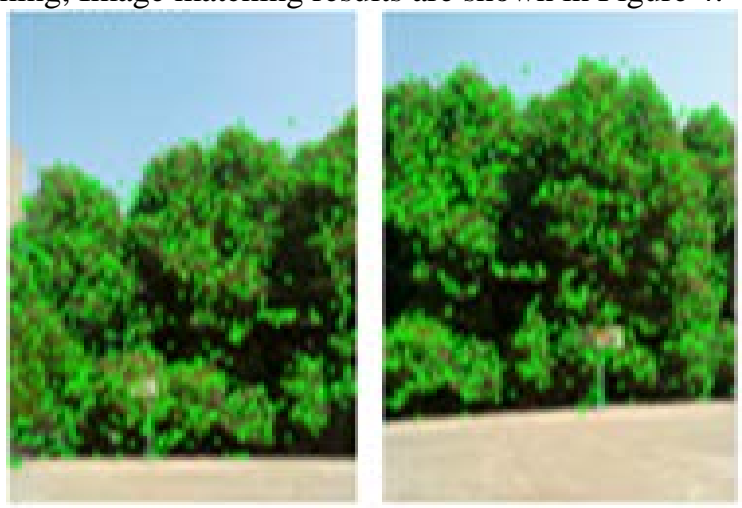

Fig2Feature extraction results 


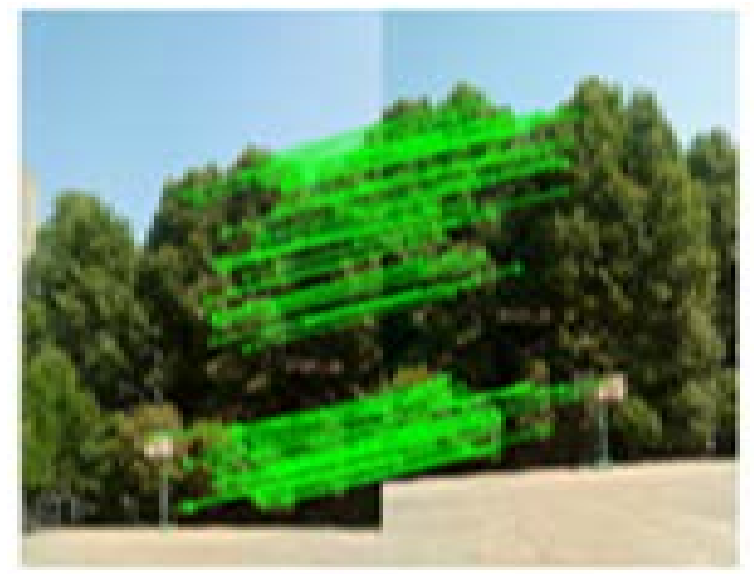

Fig3The coarse matching results

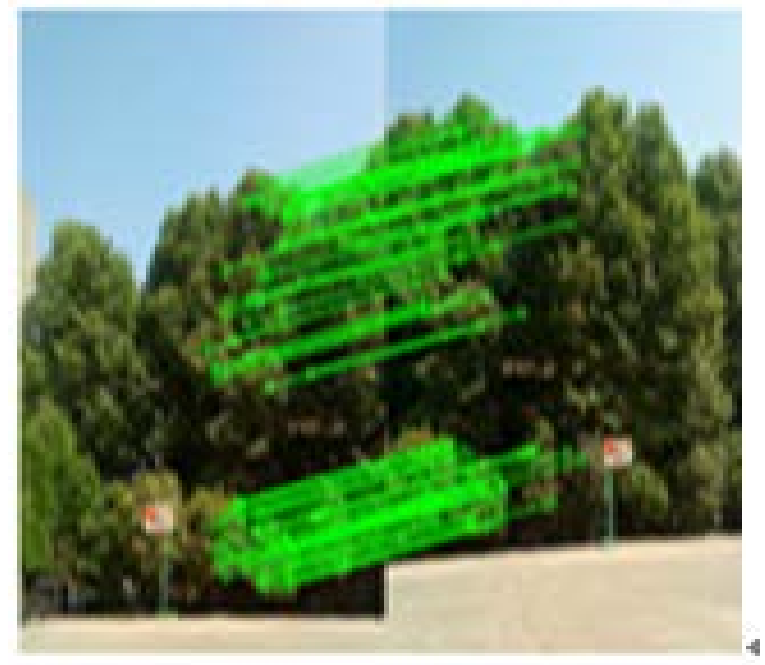

Fig4 Fine matching results

\section{Image fusion}

The resulting in the matching process transformation matrix as the projection matrix in the splicing process, in the fusion process, after theContourlet transform is performed on two images, according to the matrixwill be an image projected onto another image, and the overlappingregion of the fusion. Fusion rules of quality has a direct effect on the result of fusion.

\subsection{Contourlet transform fusion process}

On the images of A and B image fusion process: 
( 1 ) The image A, B the method of decomposition is used Contourlet transform, highfrequency sub band and low frequency sub band.

(2)The low frequency sub band and the corresponding high frequency sub bands using the corresponding fusion rules are fused to get the high frequency sub band and the corresponding low frequency sub-band coefficient.

This paper used the fusion rule of image region based on [4].

First of all, we need to draw the image similarity in the corresponding region of a pixel is:

$$
S_{A B}(x, y)=\left|\frac{2 \sum f_{A}(x, y) f_{B}(x, y)}{\sum_{r}\left[f_{A}^{2}(x, y)+f_{B}^{2}(x, y)\right]}\right|
$$

(3) In the formula, $\mathrm{f}_{\mathrm{B}}$ represents $\mathrm{f}_{\mathrm{A}}$ image fusion, $\mathrm{R}$ said to the neighborhood as the center is usually $3 * 3$ or $5 * 5$.The $S_{A B}$ range is between 0 to 1 , the highest regional similarity is 1 , said the two picture of exactly the same image information redundancy is highest, each position of the highest regional similarity. The greater the difference between the value of $\mathrm{S}_{\mathrm{AB}}$ is smaller, the smaller the regional similarity, at this time the information redundancy is low, $\mathrm{S}_{\mathrm{AB}}$ limit value can be 0 , set the similarity threshold for alpha, alphavalue of 0.7 .

\subsection{The fusion of low frequency sub band}

According to the comparison of $\mathrm{S}_{\mathrm{AB}}$ image, adopt different rules on thelow frequency and high frequency sub bands to achieve.

For the low frequency sub-band part:

$\mathrm{S}_{\mathrm{AB}}<0.7$, the fusion coefficients selected two images of a large difference in china;

$\mathrm{S}_{\mathrm{AB}}>=0.7$, Fusion of the weighted average of the rules of the low frequency coefficient.

For the high frequency part is according to the different situation of gray correlation degree for the corresponding treatment.

$\mathrm{S}_{\mathrm{AB}}<0.7$,According to the regional dispersion relation between two images of the high frequency coefficient corresponding to the discrete degree is high as the final results.

$\mathrm{S}_{\mathrm{AB}}>=0.7$,Fusion of the weighted average of the rules of the low frequency coefficient.

To this end the fusion process.

\subsection{Fusion experimental results and analysis}

Laplasse of the Pyramid portionof theContourlet transform used in this thesis used'9-7'filter, DBF filter is adopted in the group'pkva', the nlevsparameter is set to nlevs $=[0,0,4,4,5]$, to obtain the threshold function of different subband coefficients based on the " $3 *$ sigma rule" in the decomposition process of.The experimental results are shown in Figure 5: 

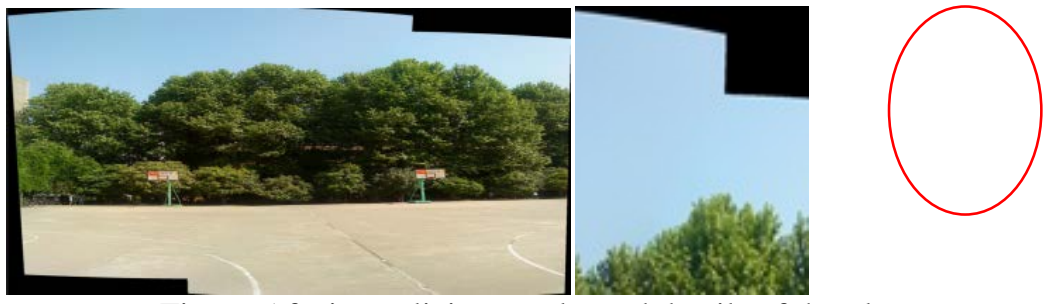

Figure 5 fusion splicing results and details of the plan

By above knowable image after fusion has been completely invisible tofusion gap, and details on the image characteristics of almost no loss.

\section{Mosaic of multiple image}

Multi image mosaic is sort of focus image. The first picture sort is determined based on the sorting process is divided into rough matching and fine matching two processes. Based on the matching ratio and threshold logarithmic time much and most compared to preliminary judgment in the coarse matching process, in the fine matching process, using the method of probability model is further judgment, to achieve image sorting. Figure 6 is the 5 picture out of order image:

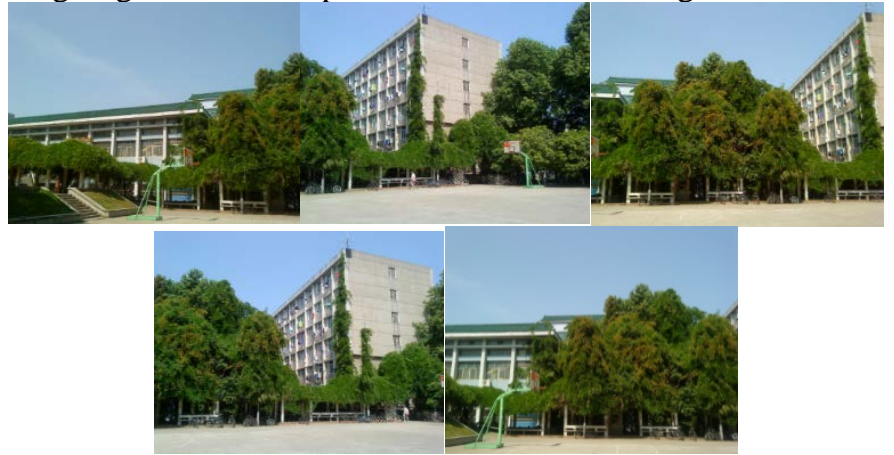

Fig. 6 pieces of disordered image

In operation, the first known image. The matching process:

Using the Harris feature point extraction method, get the number of corner feature point; After extracting the corner feature, the first image and the rest of the image is the coarse matching, matching the number of draws, the coarse matching; And then enter the fine matching stage, fine matching, find out the other image matches the first piece of image[5].Similarly, the second images to find as the first image, according to the same principle of the rest of the image matching, then can get the correct sequence images. After sorting the image sequence as shown in figure 7. 


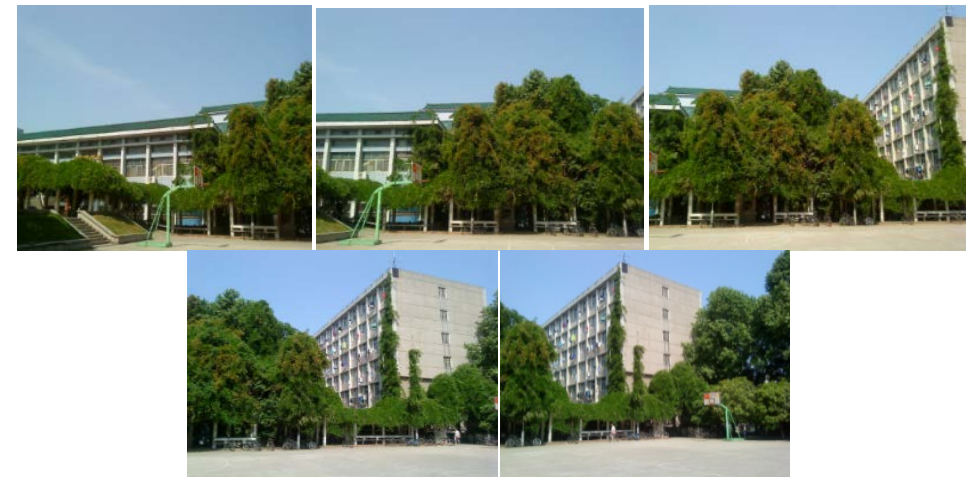

Fig. 7 image sequence sorted

Splicing method based on two images, can be multi images mosaic after the experimental results are shown in Figure 8 is obtained:

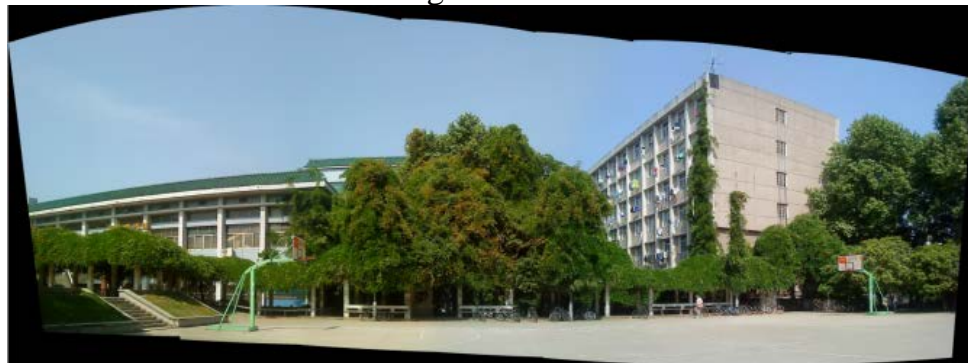

Figure 8After image stitching

Look from the effect of image stitching, no obvious mosaic trace the graphics, color is very natural, not the existence of distortion and ghosting. Objective aspect the accuracy can reach $97.4 \%$.

Therefore, through this method can get high quality image stitching.

\section{Conclusion}

In this paper, the image matching, image stitching technology of image fusion, image ranking techniques are studied and analyzed. And realize the multi images mosaic by experiment. From the known splicing effect after Contourlet transform based on image stitching technology realizes the stitching image of high quality.

\section{References}

[1] Wu Qingshuang, Fu Zhongliang. The sequence image automatic mosaic method of $[\mathrm{J}]$. computer

application of a robust. 2012,32( 2):499- 503.

[2] Wu, Yong; Yi, Pan. Cereal grain size measurement based on image processing technology [J].

[3] Xiao Fu, Wu Huizhong, Xiao Liang, Tang Yang.. Panorama 
Stitching illumination adjustment and characteristic curve based on [J]. Journal of Engineering Graphics.2005.

[4] Morimura, Atsushi; Azuma, Takeo; Uomori, Kenya. Wide dynamic range image processing technology $[\mathrm{J}]$.

[5] Feng Yuping, Deming, Sun Liyue, Zhang Wei.. Optimization design of automatic image mosaic [J]. Optics and precision engineering.2010.18 (02): 470- 475. 